\title{
Development of a superconducting bulk magnet for NMR and MRI
}

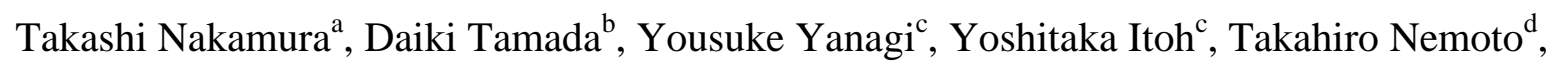 \\ Hiroaki Utumi $^{\text {d, Katsumi Kose }}$, $^{\text {a** }}$ \\ ${ }^{\text {a }}$ RIKEN, Wako 351-0198, Japan \\ ${ }^{\mathrm{b}}$ Institute of Applied Physics, University of Tsukuba, 1-1-1 Tennodai, Tsukuba 305-8573, Japan \\ ${ }^{\mathrm{c}}$ IMRA Material R\&D Co., Ltd., Kariya 448-0021, Japan \\ d JEOL Resonance, Akishima, 196-8558, Japan \\ * corresponding author. kose@bk.tsukuba.ac.jp
}

\begin{abstract}
A superconducting bulk magnet composed of six vertically stacked annular single-domain c-axis-oriented Eu-Ba-Cu-O crystals was energized to $4.74 \mathrm{~T}$ using a conventional superconducting magnet for high-resolution NMR spectroscopy. Shim coils, gradient coils, and radio frequency coils for high resolution NMR and MRI were installed in the 23 mm-diameter room-temperature bore of the bulk magnet. A 6.9 ppm peak-to-peak (PP) homogeneous region suitable for MRI was achieved in the central cylindrical region $(6.2 \mathrm{~mm}$ diameter, $9.1 \mathrm{~mm}$ length) of the bulk magnet by using a single layer shim coil. A $21 \mathrm{~Hz}$ spectral resolution that can be used for high resolution NMR spectroscopy was obtained in the central cylindrical region (1.3 mm diameter, $4 \mathrm{~mm}$ length) of the bulk magnet by using a multichannel shim coil. A clear 3D MR image dataset of a chemically fixed mouse fetus with $(50 \mu \mathrm{m})^{3}$ voxel resolution was obtained in 5.5 hours. We therefore concluded that the cryogen-free superconducting bulk magnet developed in this study is useful for high-resolution desktop NMR, MRI and mobile NMR device.
\end{abstract}

Keywords: NMR; MRI; superconducting bulk magnet; high temperature superconducting material; shim coil. 


\section{Introduction}

Superconducting magnets and permanent magnets are widely used for nuclear magnetic resonance (NMR) and magnetic resonance imaging (MRI). Because superconducting magnets can produce high and stable magnetic fields up to about $24 \mathrm{~T}$ [1], they are used for high-end NMR spectrometers and MRI systems. On the other hand, because permanent magnets need no liquid cryogen, they have been used in various applications including mobile and outdoor NMR and MRI systems [2-5]. However, permanent magnets have disadvantages in relation to the field strength (upper limit about $2 \mathrm{~T}$ [6]) and stability of the magnetic field.

In recent years, with rapidly increasing costs and possible future shortage of liquid helium, several approaches to superconducting NMR magnets with no liquid helium have been proposed [7-11]. These include high critical temperature superconductors (HTSs) such as Y-Ba-Cu-O [7, 8], $\mathrm{Bi}-\mathrm{Sr}-\mathrm{Ca}-\mathrm{Cu}-\mathrm{O}$ [9], and $\mathrm{MgB}_{2}$ [10]. The magnets made of HTS materials can be divided into wire and bulk magnets.

Like low critical-temperature superconductors (LTSs) such as $\mathrm{NbTi}$ and $\mathrm{Nb}_{3} \mathrm{Sn}$, tapes and wires of HTS materials have been developed for superconducting magnets [7-11]. However, because it is very difficult to make superconducting junctions using HTS materials, superconducting magnets using HTS wires or tapes need power sources. Therefore, field stability of the superconducting magnets using HTS materials is largely affected by the stability of the power sources and far less than that of the LTS magnets.

Unlike LTS materials, HTS materials can be used as bulk magnets because heat capacity of the HTS materials, which are mainly due to thermal lattice vibration or phonons, is much larger than that of LTS materials $[12,13]$. Such HTS bulk magnets can be energized using steady or unsteady (or pulsed) external magnetic fields. Because there is no superconducting junction in the HTS bulk magnets, the magnetic field produced by such bulk magnets have been expected to be stable and to be used for high-resolution NMR instruments [14].

With this background, in 2007 we reported the first proton NMR signal at $2.89 \mathrm{~T}(123 \mathrm{MHz})$ using a magnetic field produced in an annular HTS bulk magnet. The linewidth of the NMR spectrum was about $228 \mathrm{kHz}(\sim 1850 \mathrm{ppm})$ [15]. The homogeneity of the HTS bulk magnets was further improved [16] and we reported the first MR images using an HTS bulk magnet in 2011 [17]. The homogeneity of the magnetic field for the MRI magnet was $37 \mathrm{ppm}$ peak-to-peak in the central cylindrical region with $6.2 \mathrm{~mm}$ diameter and $9.1 \mathrm{~mm}$ length. However, the homogeneity of the magnetic field was not sufficient for high-resolution NMR spectra and MR images. In this study, we further improved the homogeneity of the magnetic field using a newly designed superconducting bulk magnet and shim coils, clarified the magnetization process using MRI measurements, and acquired high resolution NMR spectra and MR images.

\section{Materials and methods}

\subsection{Superconducting bulk magnets and cryostat}


Figure 1 shows the superconducting bulk magnets used in this study. The bulk material consisted of c-axis oriented single-domain $\mathrm{Eu}-\mathrm{Ba}-\mathrm{Cu}-\mathrm{O}$ crystals with a superconducting transition temperature of $93 \mathrm{~K}$ [18], which was used in a previous study [17]. There were several candidate materials for the bulk magnet, Y-Ba-Cu-O, Sm-Ba-Cu-O, Eu-Ba-Cu-O, and Gd-Ba-Cu-O. Among them, we chose Eu-Ba-Cu-O because it has small relative magnetic permeability $\left(\mu_{\mathrm{r}}=1.00125\right)$ and it can provide larger crystals than other materials [14].

In the previous study, the size was identical for all the bulk magnets $(60 \mathrm{~mm}$ outer diameter (OD), $28 \mathrm{~mm}$ inner diameter (ID), and $20 \mathrm{~mm}$ height). In this study, to achieve higher magnetic field homogeneity and a smaller Meissner effect for the gradient coils [19], the dimensions were changed as follows: the two end bulk magnets had $60 \mathrm{~mm}$ OD, $28 \mathrm{~mm} \mathrm{ID}$, and $23 \mathrm{~mm}$ height, while the four inner bulk magnets had $60 \mathrm{~mm}$ OD, $36 \mathrm{~mm}$ ID, and $18.5 \mathrm{~mm}$ height. The dimension of the bulk magnets (inner diameter and thickness) was optimized using a uniaxial Bean model [20] in a uniform external magnetic field. The calculation was performed using a finite element method software tool (PHOTO-series, PHOTON, Co., Ltd., Kyoto, Japan). As shown in the figure the bulk magnets were reinforced with $5 \mathrm{~mm}$-thick aluminum rings to protect them from breaks caused by the huge electromagnetic hoop stress.

The six bulk magnets were attached to the cold stage of the cryostat (88 and $23 \mathrm{~mm}$ OD and ID, respectively) as shown in Fig.2. The bulk magnets were cooled using a pulse tube refrigerator (PT121-I/C70K, AISIN SEIKI, Kariya, JAPAN,1200 W power consumption) and the temperature of the cold stage was controlled within $\pm 0.1 \mathrm{~K}$ using a built-in PID temperature controller.

\subsection{Magnetization system}

Figure 3 shows the superconducting magnet (JRTC-300/89, JASTEC, Kobe, Japan) that used to energize the bulk magnet. The diameter of the room-temperature (RT) bore of the superconducting magnet was $89 \mathrm{~mm}$; the bottom of the magnet was $1 \mathrm{~m}$ high to enable insertion of the bulk magnet into the RT bore. The superconducting magnet was originally made for a high-resolution NMR spectrometer and had 9 channel superconducting shim coils. The cryostat of the bulk magnet and the pulse tube refrigerator were placed on a lab jack as shown in the figure.

\subsection{Shim coils}

Figure 4 shows a multichannel shim coil that was developed for acquiring high-resolution NMR spectra and a single-layer shim coil developed to acquire MR images. The multichannel shim coil included the 0th- and 1st-order z coil and other 2nd-order shim coils. The shim coil was designed according to the paper published by Romeo and Hoult [21]. The coils were wound using 0.32 mm-diameter polyurethane (PU)-coated $\mathrm{Cu}$ wire. The OD and ID of the multichannel shim coil were 22 and $17 \mathrm{~mm}$, respectively. The total length of the shim coil set was about $45 \mathrm{~mm}$.

The single-layer shim coil was designed using circular base functions to approximate the inhomogeneous magnetic field that compensated the measured inhomogeneous magnetic field [22, 23]. An electric current wire pattern was calculated using the stream function method [24] from the 
approximated electric current distribution. The single-layer shim coil was wound using 0.2 mm-diameter PU-coated $\mathrm{Cu}$ wire. The OD and ID of the shim coil were 20 and $19 \mathrm{~mm}$, respectively. The total length of the shim coil set was about $60 \mathrm{~mm}$.

\subsection{Gradient coils}

We developed three gradient coil sets for this study. For measurements of the magnetic field distribution during the magnetization process, a gradient coil set with OD and ID of 16.8 and $14.8 \mathrm{~mm}$, respectively, was developed. The transverse coils were Golay coil sets and the longitudinal coil was a Maxwell pair coil. The coils were wound using $0.3 \mathrm{~mm}$-diameter PU-coated $\mathrm{Cu}$ wire. The total length of the gradient coil set was about $50 \mathrm{~mm}$. The efficiencies of the Gx, Gy, and Gz coils were 81 $\mathrm{mT} / \mathrm{m} / \mathrm{A}, 93 \mathrm{mT} / \mathrm{m} / \mathrm{A}$, and $106 \mathrm{mT} / \mathrm{m} / \mathrm{A}$, respectively.

For NMR spectrum measurements, a gradient coil set was developed with OD and ID of 16 and $12.7 \mathrm{~mm}$, respectively. The design of these coils was the same as that for the first coil set. The coils were wound using $0.25 \mathrm{~mm}$-diameter PU-coated $\mathrm{Cu}$ wire. The total length of the gradient coil set was about $45 \mathrm{~mm}$.

For high-resolution MR imaging, a gradient coil set was designed using a target field method that included the Meissner effect of the superconducting bulk magnet [19]. The Meissner effect was considered using the finite difference method. The transverse coils were made of $0.1 \mathrm{~mm}$-thick $\mathrm{Cu}$ foil and the longitudinal coil was wound using $0.2 \mathrm{~mm}$-diameter PU-coated $\mathrm{Cu}$ wire. The OD and ID of the gradient coils were 22 and $20 \mathrm{~mm}$, respectively. The total length of the gradient coil set was about $55 \mathrm{~mm}$. The efficiencies of the Gx, Gy, and Gz coils were $53 \mathrm{mT} / \mathrm{m} / \mathrm{A}, 53 \mathrm{mT} / \mathrm{m} / \mathrm{A}$, and $74 \mathrm{mT} / \mathrm{m} / \mathrm{A}$, respectively.

\section{5. $R F$ coils}

We used several saddle-shaped and solenoid RF coils for measurements of NMR spectra and MR images. For measurements of the magnetic field distribution during the magnetization process, we used a one-turn saddle-shaped coil (12 mm diameter, $20 \mathrm{~mm}$ length) made of $0.1 \mathrm{~mm}$ thick $\mathrm{Cu}$ foil. For NMR spectrum measurements, a five-turn solenoid coil (2.5 mm ID, $4 \mathrm{~mm}$ length) wound using $0.5 \mathrm{~mm}$-diameter PU-coated $\mathrm{Cu}$ wire was used. For measurement of MR images of a chemically fixed mouse fetus, a four-turn solenoid coil (6 mm ID, $6 \mathrm{~mm}$ length) was used. All the RF coil circuits were matched to $202 \mathrm{MHz}$ using a vector network analyzer.

\subsection{Spectrometer electronics}

We used an NMR spectrometer (ECX-400P, JEOL, Akishima, Japan) for NMR spectrum measurements and an MRI transceiver (DTRX4-200MHz, MRTechnology, Tsukuba, Japan) for measurements of MR images. For MR images, we used home-built gradient drivers ( $\pm 10 \mathrm{~V}, \pm 5 \mathrm{~A})$ incorporating power operational amplifiers (PA-12, Apex Microtechnology, Tucson, Arizona).

\subsection{Measurements of magnetic field distribution}


The magnetic field distribution in the bulk magnet was measured using $\mathrm{CuSO}_{4}$-doped water in an NMR sample tube with OD and ID of 8 and $6.9 \mathrm{~mm}$, respectively. The pulse sequences used were a pair of 3D spin-echo imaging sequences (70 ms repetition time (TR), $20 \mathrm{~ms}$ echo time (TE),

image matrix $64^{3}$, field of view $(12.8 \mathrm{~mm})^{3}$, total scan time 4.8 minutes). Between the two spin-echo pulse sequences, only the timing of the $180^{\circ}$ refocusing pulse differed by $0.3 \mathrm{~ms}$ to measure the phase shift of nuclear magnetization caused by the inhomogeneous magnetic field. The magnetic field distribution in the central cylindrical region of $6 \mathrm{~mm}$ diameter and $6 \mathrm{~mm}$ length was evaluated for the magnetization process.

\subsection{Magnetization of the superconducting bulk magnet}

The bulk magnet was magnetized as follows. The bulk magnet was inserted into the center of the RT bore of the superconducting magnet and the magnetic field of the superconducting magnet was raised to $4.74 \mathrm{~T}$. The bulk magnet was cooled to $100 \mathrm{~K}$. The magnetic field of the superconducting magnet was shimmed using the 1st-order superconducting shim coils ( $\mathrm{x}, \mathrm{y}, \mathrm{z})$. The shimming was monitored by the NMR signal of $\mathrm{CuSO}_{4}$-doped water in an $8 \mathrm{~mm}$-diameter NMR sample tube inserted into the saddle-shaped RF coil placed in the bore of the bulk magnet. After the shimming was completed, the shim coil circuits were closed to establish a persistent current mode. After the magnetic field distribution was measured using echo time-shifted 3D spin-echo sequences, as described later, the bulk magnet was further cooled to $92 \mathrm{~K}$ and the magnetic field distribution was measured in the same way. After the temperature of the bulk magnet was further cooled to $84,77,60$, and $50 \mathrm{~K}$, the magnetic field distribution measurements were repeated.

After the magnetic field distribution was measured at $50 \mathrm{~K}$, the electric current of the main magnet was slowly decreased to $4.0 \mathrm{~T}$ at a rate of $-24 \mathrm{mT} / \mathrm{h}$. The magnetic field distribution was measured in the same way, as described later. After the magnetic field was further decreased to 3.0, 2.0, 1.0, and $0.0 \mathrm{~T}$, the magnetic field distribution measurements were repeated.

\section{Results}

\subsection{Magnetization process}

Figure 5 shows the magnetic field distribution measured during the cooling process of the bulk magnet. The magnetic field inhomogeneity decreased monotonously from 7.2 to $4.5 \mathrm{ppm}$ peak-to-peak $(-2.7 \mathrm{ppm})$ and the central resonance frequency increased monotonously from 202.080 to $202.134 \mathrm{MHz}$ (+264 ppm) from 100 to $50 \mathrm{~K}$.

Figure 6 shows the magnetic field distribution measured during the demagnetization process of the superconducting magnet from 4.74 to $0.0 \mathrm{~T}$. The magnetic field inhomogeneity increased monotonously from 4.5 to $27.9 \mathrm{ppm}$ peak-to-peak $(+23.4 \mathrm{ppm})$ and the central resonance frequency decreased monotonously from 202.134 to $202.051 \mathrm{MHz}$ (-413 ppm).

\subsection{Magnetic field shimming}


Figure 7 shows the NMR spectra of ethanol measured in the bulk magnet. The ethanol sample was filled with a glass tube (OD and ID $2.3 \mathrm{~mm}$ and $1.3 \mathrm{~mm}$, respectively, $10 \mathrm{~mm}$ length), and sealed with epoxy resin. The lowest spectrum was measured with no shim current. The full width at half maximum (FWHM) of the spectrum was about $3 \mathrm{kHz}(15 \mathrm{ppm})$. The middle spectrum was measured with optimum currents for the 1st order shim (gradient) coils. The FWHM of the spectrum was about $100 \mathrm{~Hz}(0.5 \mathrm{ppm})$. The upper spectrum was measured with optimum currents for the 1st order and multichannel shim coils. The FWHM of the spectrum was $21 \mathrm{~Hz}(0.1 \mathrm{ppm})$.

Figure 8 shows the magnetic field distribution measured when the electric current for the single-layer shim coil was 0 and $600 \mathrm{~mA}$. The magnetic field inhomogeneity improved from 42.9 to $6.9 \mathrm{ppm}$ peak-to-peak for the central cylindrical region with $6.2 \mathrm{~mm}$ diameter and $9.1 \mathrm{~mm}$ length, which is 5 times better than in the previous study [13]. Because the theoretically predicted optimum current value was $550 \mathrm{~mA}$, the magnetic field distribution was measured repeatedly by varying the current value in $50 \mathrm{~mA}$ steps. The experimentally determined optimum value for the shim coil current was $600 \mathrm{~mA}$.

Figure 9 shows cross-sectional images selected from a 3D image dataset of a formalin-fixed mouse fetus (Jcl:ICR mouse, 14 days post conception) acquired with a 3D spin-echo sequence (TR $200 \mathrm{~ms}$, TE $20 \mathrm{~ms}$, image matrix $256 \times 128 \times 96$, voxel size $(50 \mu \mathrm{m})^{3}$, number of excitations 8 , total measurement time $5.5 \mathrm{~h}$ ). Image distortion and signal loss were not evident.

\section{Discussion}

\subsection{Magnetization process of the bulk magnet}

As shown in the cooling process of the bulk magnet (Fig.5), the magnetic field strength in the bore of the bulk magnet increased considerably (+264 ppm) and the magnetic field inhomogeneity in the bulk magnet decreased slightly $(-2.7 \mathrm{ppm})$ when the bulk magnet was cooled from $100 \mathrm{~K}$ to 50 $\mathrm{K}$ in a homogeneous magnetic field of $4.74 \mathrm{~T}$.

We have already observed similar increase of the magnetic field strength (NMR resonance frequency) for a bulk magnet using $\mathrm{Gd}-\mathrm{Ba}-\mathrm{Cu}-\mathrm{O}$ material [14]. However, the increase of the NMR resonance frequency for this bulk magnet was $10-50 \mathrm{kHz}(50-250$ ppm), dependent on the run of experiments, which is less than or equal to that of $\mathrm{Eu}-\mathrm{Ba}-\mathrm{Cu}-\mathrm{O}$ $(250-300 \mathrm{ppm})$ material, although the relative magnetic permeability $\left(\mu_{\mathrm{r}}\right)$ of the $\mathrm{Gd}-\mathrm{Ba}-\mathrm{Cu}-\mathrm{O}$ material at $50-95 \mathrm{~K}$ was $1.019-1.012$, which had larger paramagnetic moment effect comparing with Eu-Ba-Cu-O $\left(\mu_{\mathrm{r}}=1.00125\right.$, at $\left.50-95 \mathrm{~K}\right)$ material [14]. Therefore, we think this increase of the magnetic field (+264 ppm in this experiment) is not caused by the paramagnetic effect of the material but caused by superconducting property of the material.

We therefore think that one possible mechanism of the increase of the magnetic field in the bore of the magnet is that magnetic flux penetrating the bulk material above Tc $(93 \mathrm{~K})$ was pushed to the bore of the magnet because the lower critical field $\left(\mathrm{H}_{\mathrm{cl}}\right)$ of the $\mathrm{Eu}-\mathrm{Ba}-\mathrm{Cu}-\mathrm{O}$ material increases with decreasing temperature. 
As shown in the demagnetization process of the superconducting magnet (Fig.6), the magnetic field strength in the bore of the bulk magnet decreased considerably ( $-413 \mathrm{ppm})$ and the magnetic field inhomogeneity in the bulk magnet increased slightly $(+23.4 \mathrm{ppm})$ when the external magnetic field decreased from $4.74 \mathrm{~T}$ to $0 \mathrm{~T}$ at $50 \mathrm{~K}$. This experimental result suggests that superconducting current was induced in the bulk magnet and magnetic flux tubes were pinned in the bulk material to keep the total magnetic flux in the bore of the bulk magnet. Although there are many factors affecting the magnetic field inhomogeneity, such as imperfection, micro crack, and temperature distribution of the bulk material, the inhomogeneity can be compensated using several techniques used in high-resolution NMR spectroscopy because the order of the inhomogeneity is a few tens of ppm.

\subsection{Magnetic field shimming}

The magnetic field inhomogeneity after the bulk magnet was energized was about $28 \mathrm{ppm}$ for the central cylindrical region (6 $\mathrm{mm}$ diameter, $6 \mathrm{~mm}$ length), which is suitable value to compensate using resistive shim coils. For MR imaging other than chemical shift imaging, magnetic field inhomogeneity on the order of several ppm is sufficient because diamagnetic biological samples usually produce inhomogeneous magnetic field of several ppm. To achieve this homogeneity, the single layer shim coil is useful because the magnetic field inhomogeneity was improved from 42.9 to 6.9 ppm by using this shim coil.

On the other hand, for high resolution NMR spectroscopy, magnetic field inhomogeneity on the order of a few $\mathrm{Hz}$ or $0.01 \mathrm{ppm}$ is required. To achieve this homogeneity, multi-channel shim coil, sample spinning, and susceptibility matched RF coil are required. Therefore, although further improvements are required for high resolution NMR spectroscopy, we think we are now approaching to the goal.

\section{Conclusion}

We energized a superconducting bulk magnet to $4.74 \mathrm{~T}$ using a conventional superconducting magnet for high-resolution NMR. We achieved magnetic field inhomogeneity of 6.9 ppm peak-to-peak in the central cylindrical region of $6.2 \mathrm{~mm}$ diameter and $9.1 \mathrm{~mm}$ length of the bulk magnet using an single layer shim coil. We also achieved $21 \mathrm{~Hz}$ or $0.1 \mathrm{ppm}$ spectral resolution in the central cylindrical region of $1.3 \mathrm{~mm}$ diameter and $4 \mathrm{~mm}$ length of the bulk magnet by using a multichannel shim coil. We thus conclude that our bulk magnet is sufficiently good for MR microimaging and not good for high-resolution NMR spectroscopy at present, but promising if higher-order shimming, a sample spinning unit, and a susceptibility matched RF coil are successfully installed.

\section{Acknowledgement}

This study was supported by a Development of Systems and Technologies for Advanced Measurement and Analysis project by Japan Science and Technology Agency. 


\section{References}

[1] K. Hashi, S. Ohki, S. Matsumoto, G. Nishijima, A. Goto, K. Deguchi, K. Yamada, T. Noguchi, S. Sakai, M. Takahashi, Y. Yanagisawa, S. Iguchi, T. Yamazaki, H. Maeda, R. Tanaka, T. Nemoto, H. Suematsu, T. Miki, K. Saito, T. Shimizu, Achievement of 1020 MHz NMR, J. Magn. Reson. 256 (2015) 30-33.

[2] K. Kose, Compact MRI for Chemical Engineering, in: S. Stapf, S. Han (Eds.), NMR in Chemical Engineering, Wiley-VCH, Weinheim, (2006) 77-89.

[3] B. Blu $\square$ mich, S.J. Appelt, F. Casanova, NMR at low magnetic fields, Chem. Phys. Lett. 477 (2009) 231-240.

[4] J. Mitchell, L.F. Gladden, T.C. Chandrasekera, E.J. Fordham, Low-field permanent magnets for industrial process and quality control, Progress in Nuclear Magnetic Resonance Spectroscopy 76 (2014) $1-60$.

[5] S.S. Zalesskiy, E. Danieli, B. Blu $\square$ mich, V.P. Ananikov, Miniaturization of NMR Systems: Desktop Spectrometers, Microcoil Spectroscopy, and "NMR on a Chip" for Chemistry, Biochemistry, and Industry, Chem. Rev. 114 (2014) 5641-5694.

[6] T. Haishi, M. Aoki, E. Sugiyama, Development of a 2.0 Tesla permanent magnetic circuit for NMR/MRI, Proc. Int. Soc. Mag. Reson. Med. 13 (2005) 869.

[7] B.J. Parkinson, R. Slade, M.J.D. Mallett, V. Chamritski. Development of a cryogen free $1.5 \mathrm{~T}$ YBCO HTS magnet for MRI. IEEE Trans. Appl. Supercond., 23 (2013) 4400405.

[8] R.A. Slade, B.J. Parkinson, R.M. Walsh, Test Results for a 1.5 T MRI System Utilizing a Cryogen-free YBCO Magnet, IEEE Trans. Appl. Supercond., 24 (2014) 4400705.

[9] Y. Terao, O. Ozaki, C. Ichihara, S. Kawashima, T. Hase, H. Kitaguchi, S. Kobayashi, K. Sato, I. Nakajima, N. Oonishi, M. Poole, K. Takeda, S. Urayama, and H. Fukuyama . Newly designed 3 T 
MRI magnet wound with Bi-2223 tape conductors. IEEE Trans. Appl. Supercond., 23 (2013) 4400904.

[10] M. Razeti, S. Angius, L. Bertora, D. Damiani, R. Marabotto, M. Modica, D. Nardelli, M. Perrella, and M. Tassisto, Construction and operation of cryogen free $\mathrm{MgB}_{2}$ magnets for open MRI systems, IEEE Trans. Appl. Supercond., 18 (2008) 882-886.

[11] H. Maeda, Y. Yanagisawa. Recent developments in high-temperature superconducting magnet technology (review). IEEE Trans. Appl. Supercond., 24 (2014) 1-12.

[12] S. Nariki, N. Sakai, M. Murakami, Processing of high-performance Gd-Ba-Cu-O bulk superconductor with Ag addition. Supercond. Sci. Technol. 15 (2002) 648-652.

[13] M. Tomita, M. Murakami, High-temperature superconductor bulk magnets that can trap magnetic fields of over 17 tesla at $29 \mathrm{~K}$, Nature, 421 (2003) 517-520.

[14] T. Nakamura, Y. Itoh, M. Yoshioka, N. Sakai, S. Nariki, I. Hirabayashi, H. Utsumi, Application of a Compact Cryogen-free Superconducting Bulk Magnet to NMR - Evaluation of a Superconducting Bulk Magnet using NMR Signal -, Teion Kogaku 48 (2011) 139-148 (in Japanese).

[15] T. Nakamura, M. Yoshikawa, Y. Itoh, H. Koshino, Development of a Superconducting Magnet for Nuclear Magnetic Resonance Using Bulk High-Temperature Superconducting Materials, Concept Magn. Reson. B (Magn. Reson. Eng.) 31B (2007) 65.

[16] N. Sakai, S. Nariki, E. Teshima, M. Morita, I. Hirabayashi, T. Nakamura, Y. Itoh, M. Yoshikawa, H. Utsumi, Teion Kogaku 46 (2011) 131-138 (in Japanese).

[17] K. Ogawa, T. Nakamura, Y. Terada, K. Kose, T. Haishi, Development of a magnetic resonance microscope using a high Tc bulk superconducting magnet, Appl. Phys. Lett. 98 (2011) 234101.

[18] S. Nariki, N. Sakai, I. Hirabayashi, M. Yoshikawa, Y. Itoh, T. Nakamura, H. Utumi, Preparation and properties of Eu-Ba-Cu-O superconductors, Physica C 468 (2008) 1451-1455.

[19] D. Tamada, T. Nakamura, Y. Ito, K. Kose, Experimental evaluation of the magnetization process in a high Tc bulk superconducting magnet using magnetic resonance imaging, Physica C 492 (2013) $174-177$. 
[20] C. P. Bean, Magnetization of hard superconductors, Phys. Rev. Lett. , 8 (1962) 250-253.

[21] F. Romeo, D. I. Hoult, Magnetic field profiling: Analysis and Correcting Coil Design, Magn. Reson. Med. 1 (1984) 44-65.

[22] R. Shigeki and K. Kose, A Single-Channel Planar Shim Coil for a Permanent Magnet, Proc. Int. Soc. Magn. Reson. Med. 19 (2010) 1542.

[23] D. Tamada, K. Kose, T. Haishi, A New Planar Single-Channel Shim Coil Using Multiple Circular Currents for Magnetic Resonance Imaging, Appl. Phys. Express 5 (2012) 056701.

[24] R. Turner, Gradient coil design: a review of methods. Magn. Reson. Imaging, 11 (1993) 903-920.

[25] D. Tamada, T. Nakamura, K. Kose, A gradient coil design for a high-temperature superconducting bulk magnet using the finite-difference method, Supercond. Sci. Technol. 28 (2015) 095010.

Figure captions

Fig. 1. The bulk superconductors used for the superconducting bulk magnet. The material of the bulk magnets is c-axis oriented $\mathrm{EuBa}_{2} \mathrm{Cu}_{3} \mathrm{O}_{\mathrm{y}}$ crystals $\left(\mathrm{T}_{\mathrm{c}}=93 \mathrm{~K}\right)$. The OD of all the bulk magnets is 60 $\mathrm{mm}$; the ID and thickness of the two end magnets (front right and back left) are 28 and $23 \mathrm{~mm}$, respectively; the ID and thickness of the other four magnets are 36 and $18.5 \mathrm{~mm}$, respectively. The bulk magnets are inserted in $5 \mathrm{~mm}$-thick aluminum rings to resist hoop stress.

Fig. 2. Cryostat for the superconducting bulk magnet. The stacked bulk magnets are attached on a cold stage cooled with a pulse tube refrigerator. The OD and RT bore of the cryostat are 88 and $23 \mathrm{~mm}$, respectively.

Fig. 3. Magnetization system for the superconducting bulk magnet. The superconducting magnet used to energize the bulk magnet is shown to the left of the bulk magnet. The leg of the superconducting magnet was elongated to $1 \mathrm{~m}$ for the bulk magnet. The bulk magnet and the cooling system are placed on a lab jack that can be moved up and down as shown in the right-hand image. The radius of the 5 Gauss line is about $0.5 \mathrm{~m}$.

Fig. 4. RTshim coils developed for the bulk superconducting magnet. (a) A multi-channel shim coil system developed for NMR spectrum acquisition. The OD and ID are 22 and $17 \mathrm{~mm}$, respectively. The diameter of the PU-coated $\mathrm{Cu}$ wire is $0.32 \mathrm{~mm}$. (b) A single-layer shim coil developed for MR 
image acquisition. The OD and ID are 20 and $19 \mathrm{~mm}$, respectively. The diameter of the PU-coated $\mathrm{Cu}$ wire is $0.2 \mathrm{~mm}$.

Fig. 5. (a) Magnetic field distribution measured during the cooling process in the $4.74 \mathrm{~T}$ magnetic field for the central cylindrical region $(6 \mathrm{~mm}$ diameter, $6 \mathrm{~mm}$ length). (b) Magnetic field inhomogeneity and central precession frequency measured during the cooling process in the $4.74 \mathrm{~T}$ homogeneous magnetic field.

Fig. 6. (a) Magnetic field distribution measured during the demagnetization process at $50 \mathrm{~K}$ for the central cylindrical region (6 mm diameter, $6 \mathrm{~mm}$ length). (b) Magnetic field inhomogeneity and central precession frequency measured during the demagnetization process at $50 \mathrm{~K}$.

Fig. 7. NMR spectra of ethanol measured in the superconducting bulk magnet. The lower, middle, and upper spectra were measured with no shim coil current, optimum currents for the 1st order shim (gradient) coil, and optimum currents for the 1 st and multichannel shim coils, respectively.

Fig. 8. Magnetic field distribution measured when the current of the single layer shim coil is (a) $0 \mathrm{~mA}$ and (b) $600 \mathrm{~mA}$. The left- and right-hand figures show magnetic field distributions in the central yz and xy planes, respectively.

Fig. 9. Cross-sectional images selected from a 3D image dataset of a formalin fixed mouse fetus acquired with a 3D spin-echo sequence (repetition time $200 \mathrm{~ms}$, echo time $20 \mathrm{~ms}$, image matrix $256 \times 128 \times 96$, voxel size $(50 \mu \mathrm{m})^{3}$, number of excitation 8 , and total measurement time $\left.5.5 \mathrm{~h}\right)$. 

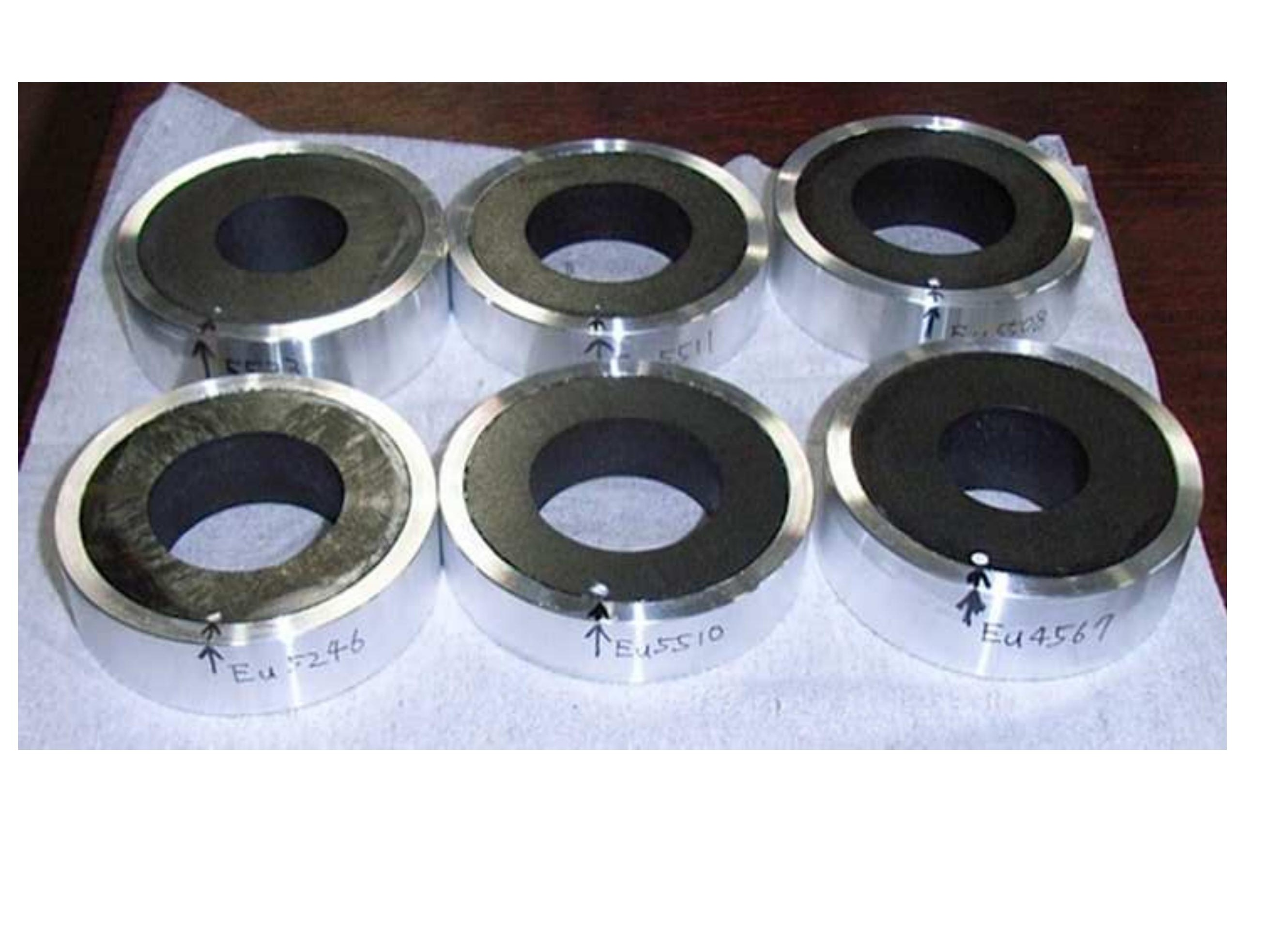

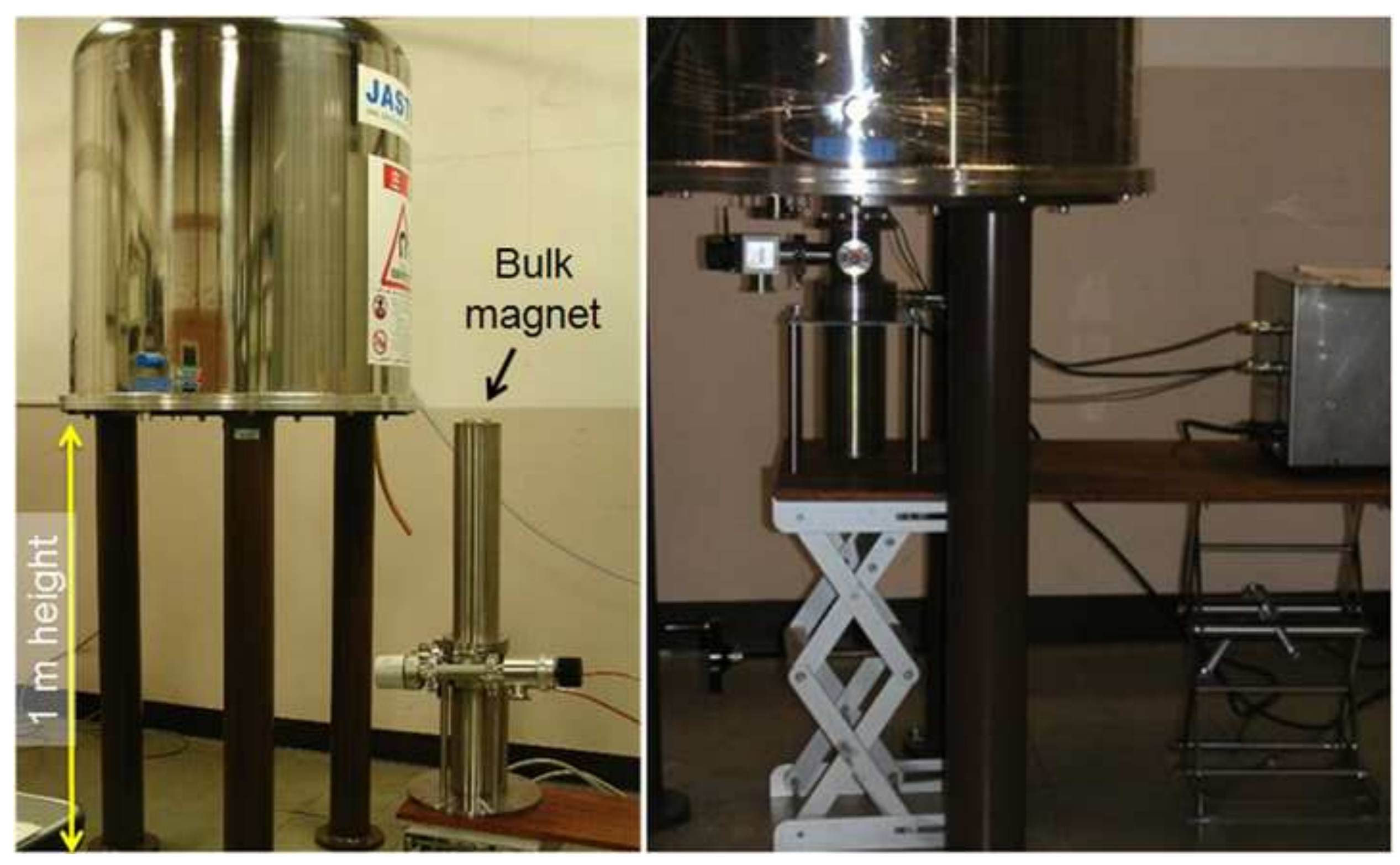

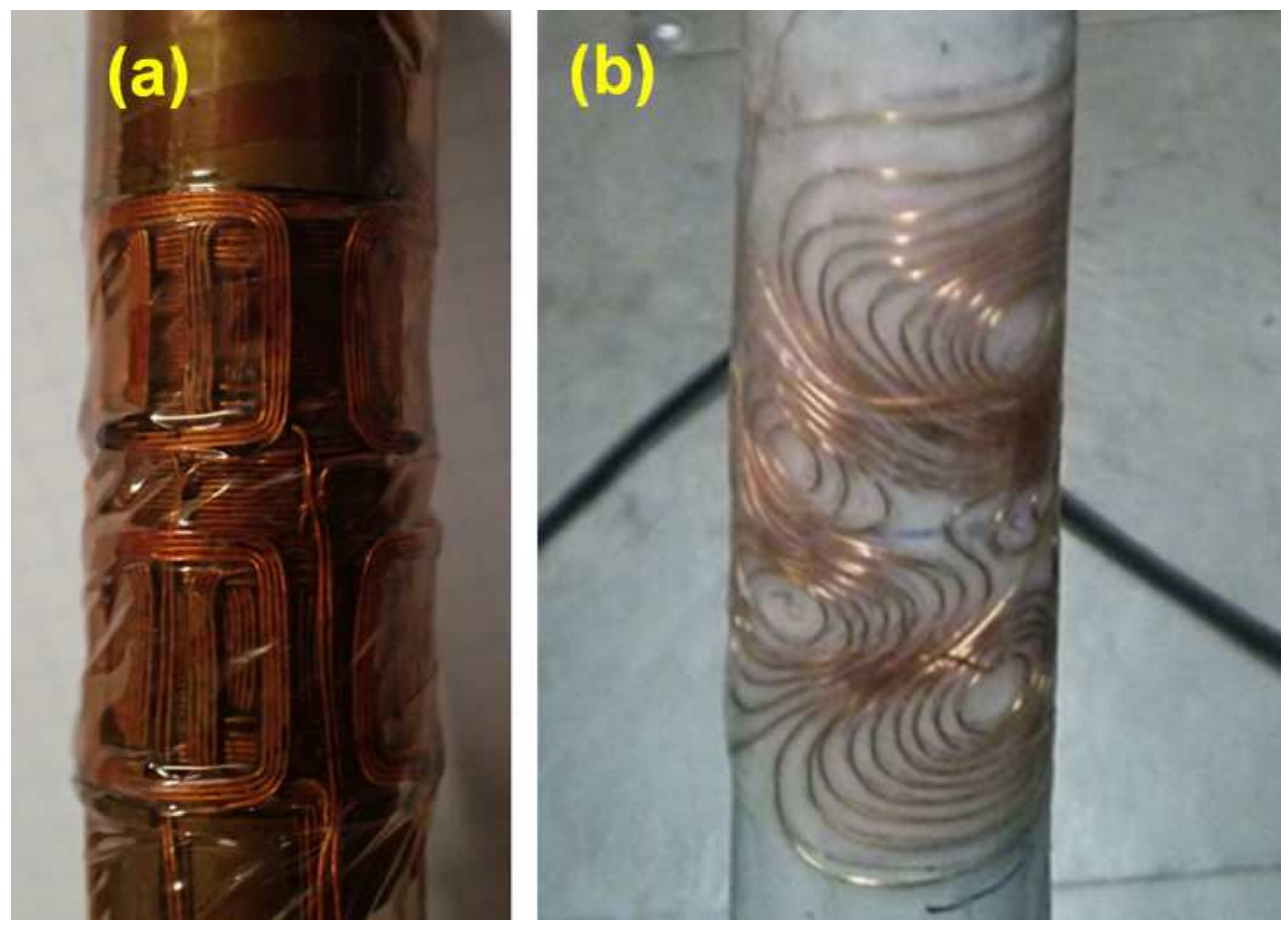
Temperature of bulk magnet

$$
T_{b}=100 \mathrm{~K}
$$

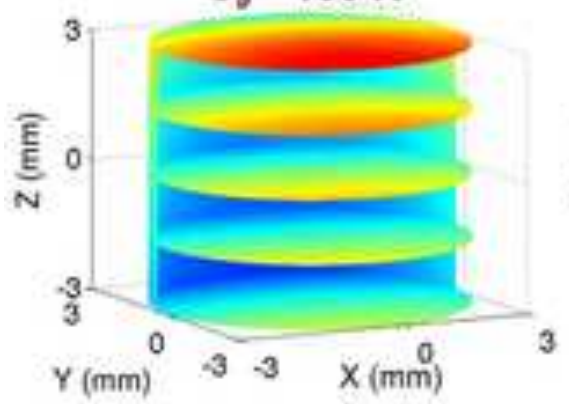

$$
T_{\mathrm{b}}=70 \mathrm{~K}
$$

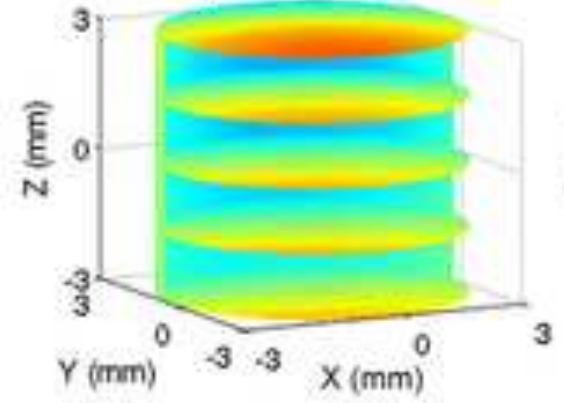

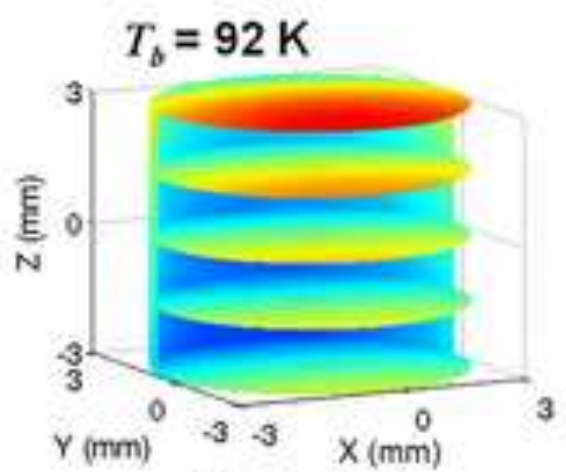

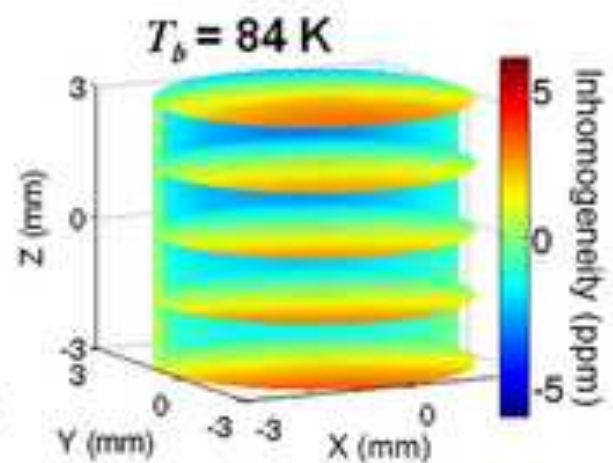

$$
T_{b}=60 \mathrm{~K}
$$
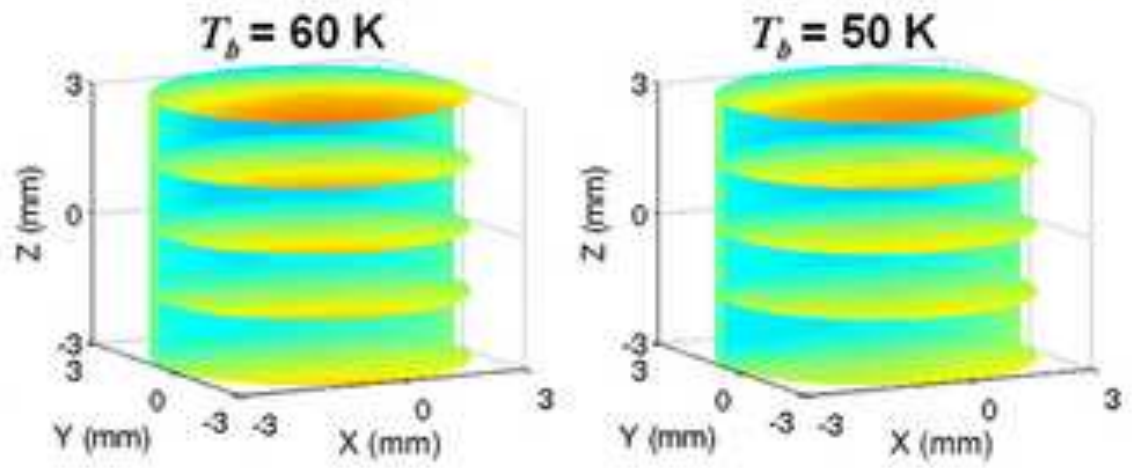

(a)

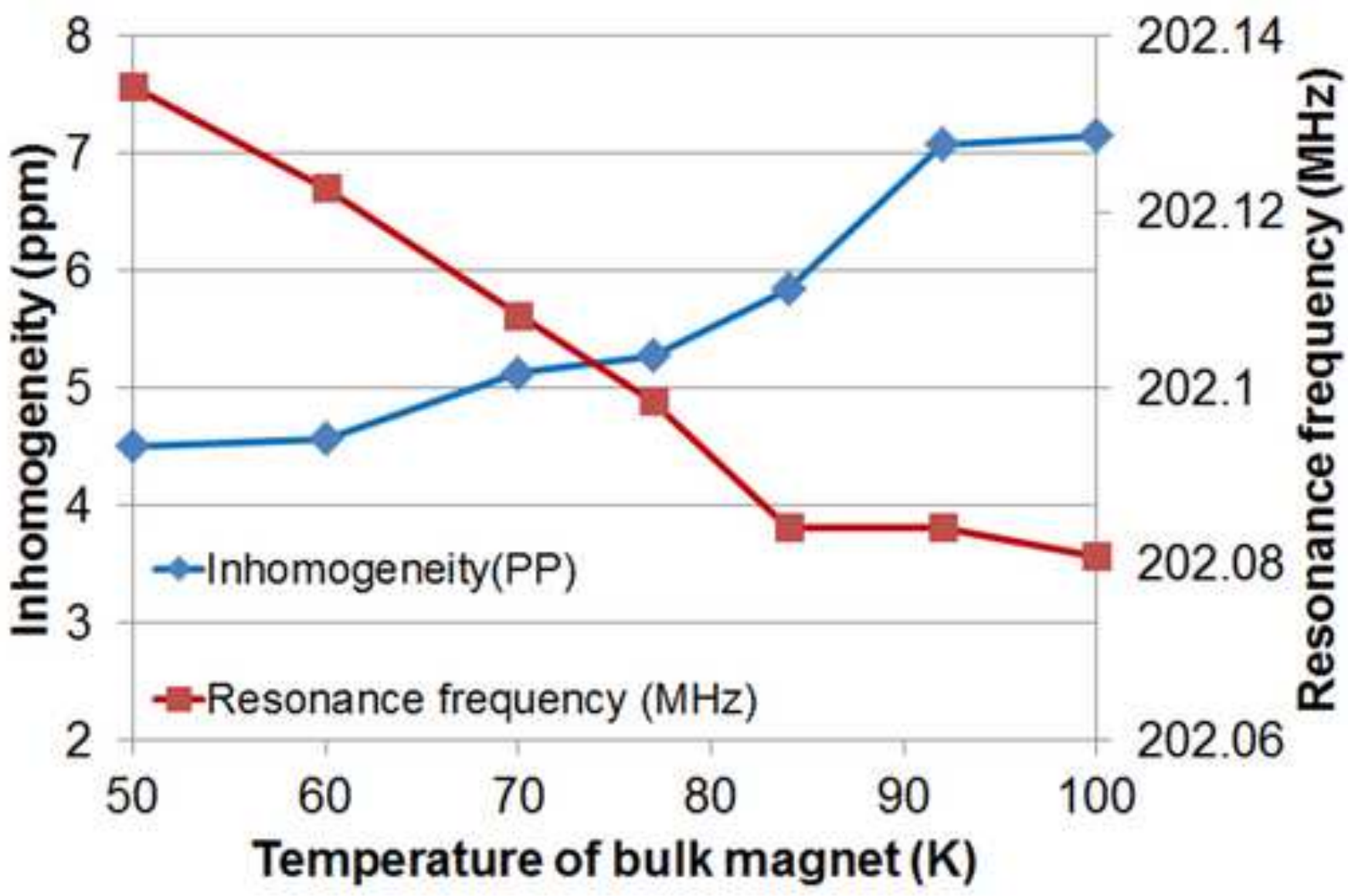

(b) 
External magnetic field strength

$B_{e}=4.74 \mathrm{~T}$
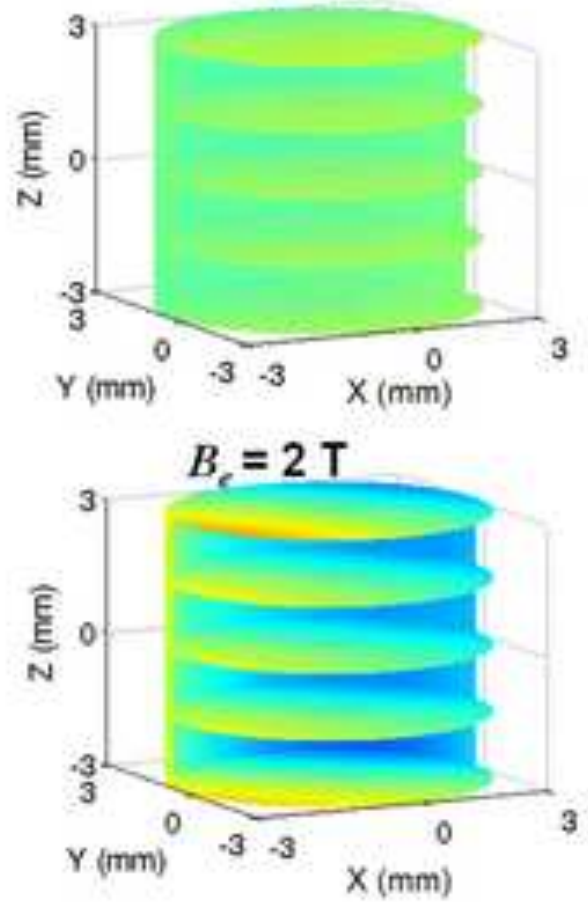
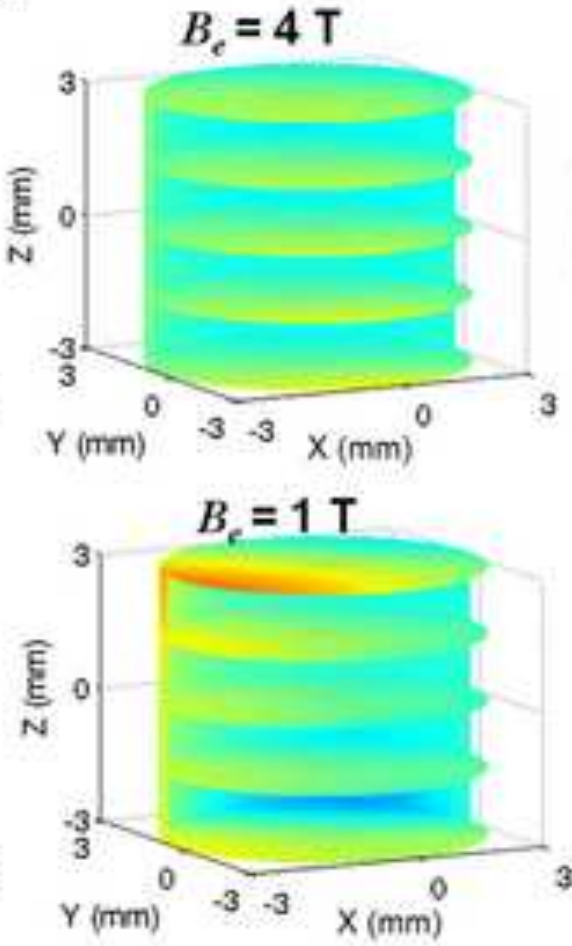

(a)
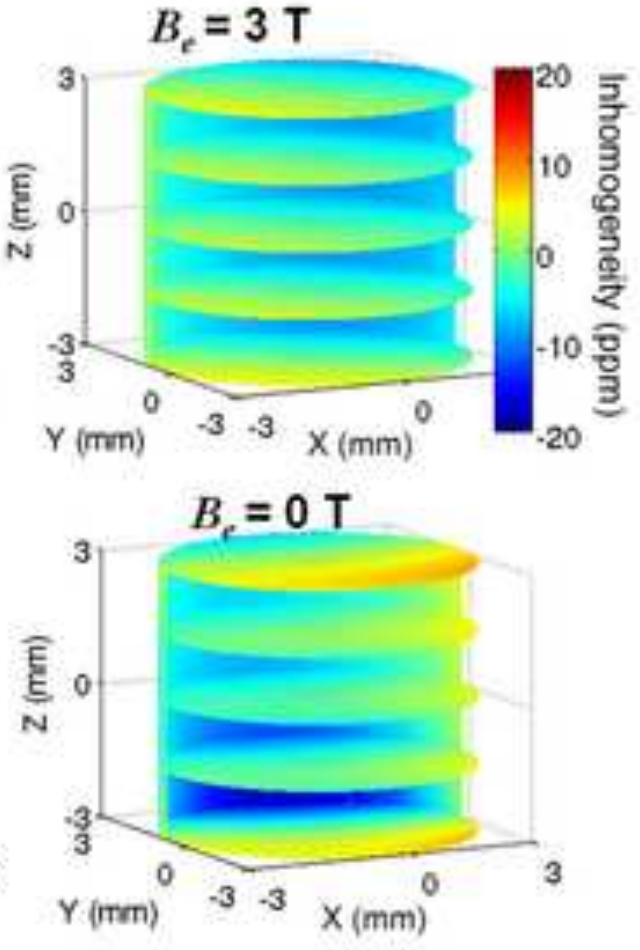

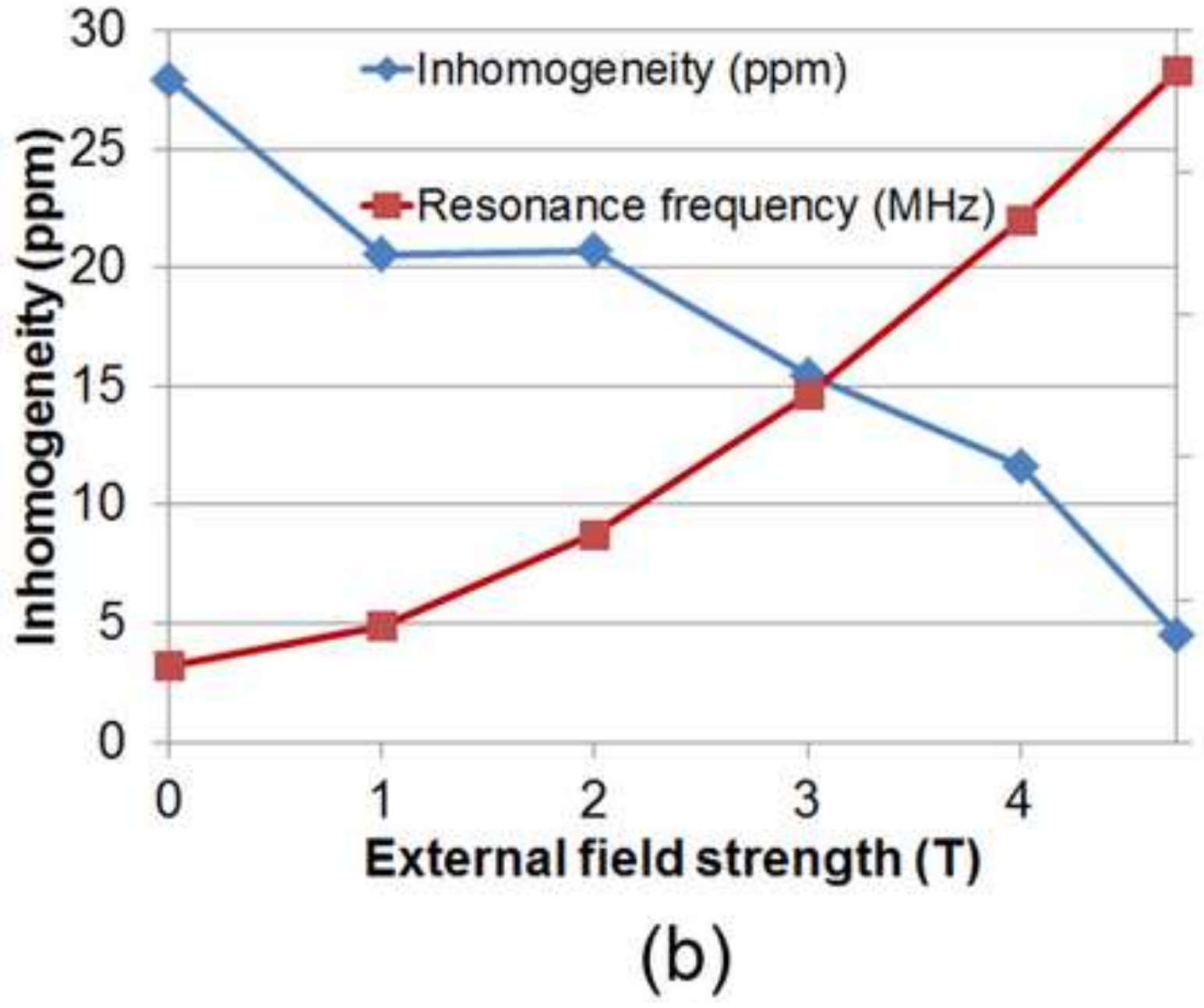

202.14

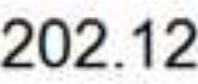

202.1 흥

202.04

202.06

202.08

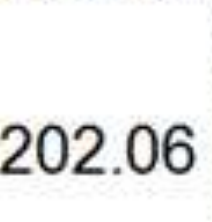

.

0 


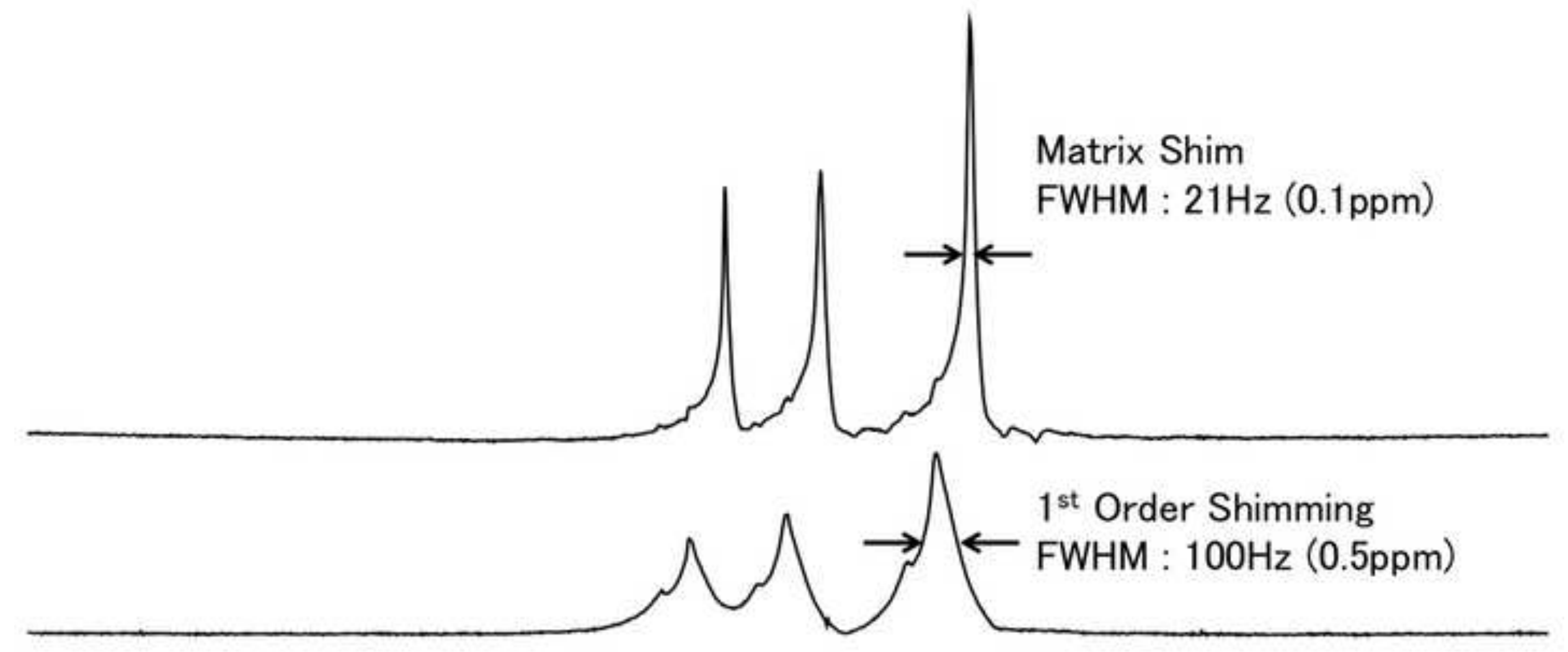

Without Shimming FWHM : $2979 \mathrm{~Hz}$ (15ppm)

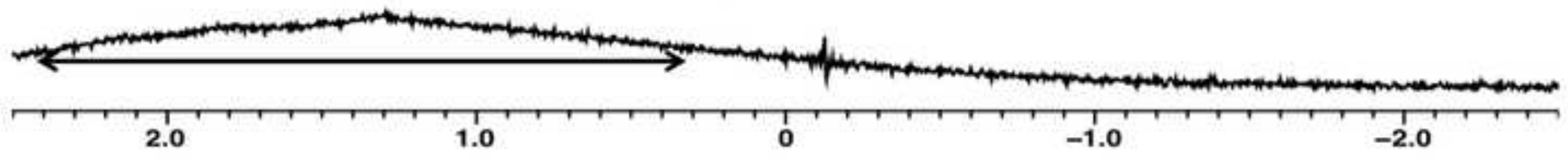

$1 \mathrm{H}$ : kilohertz 

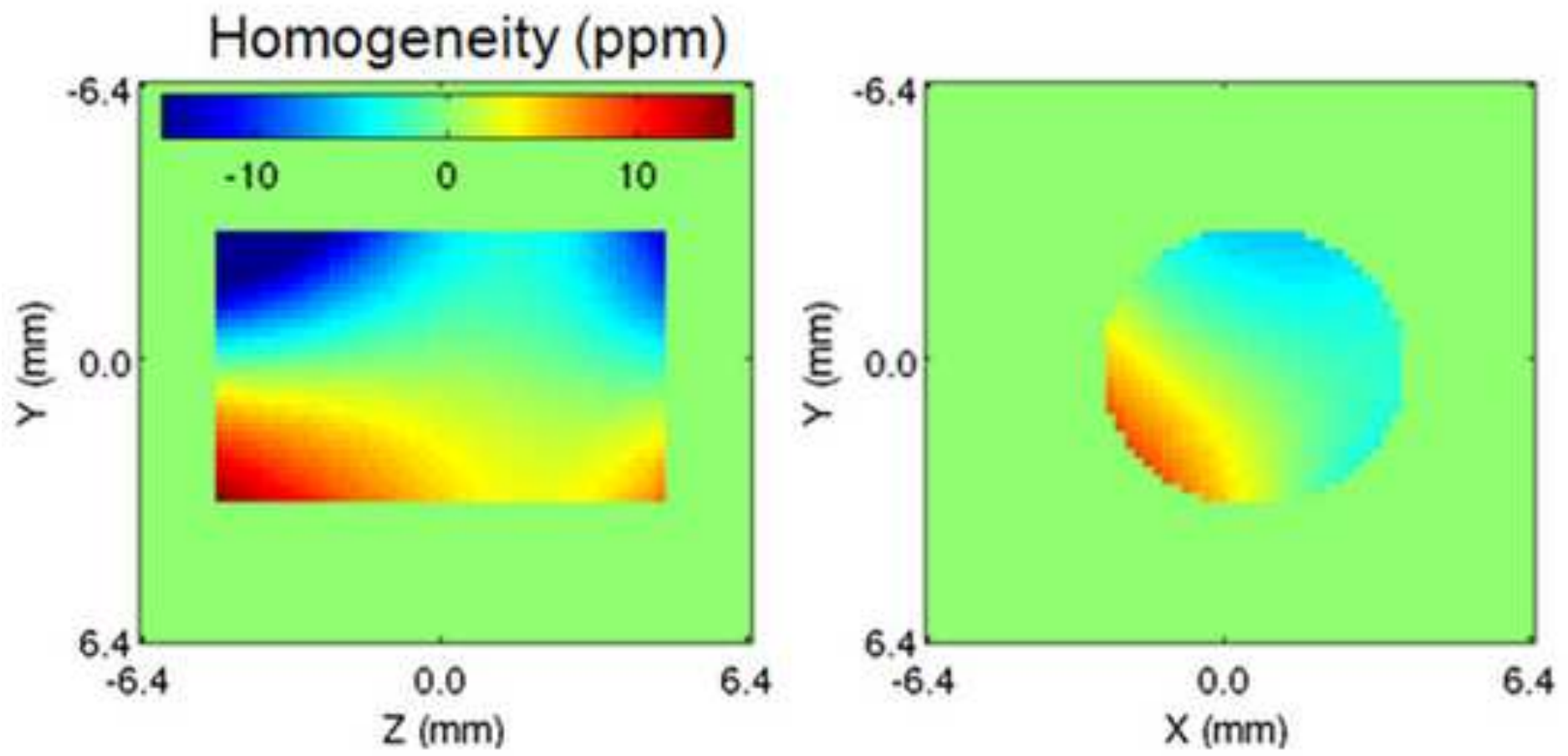

(a)

Homogeneity (ppm)
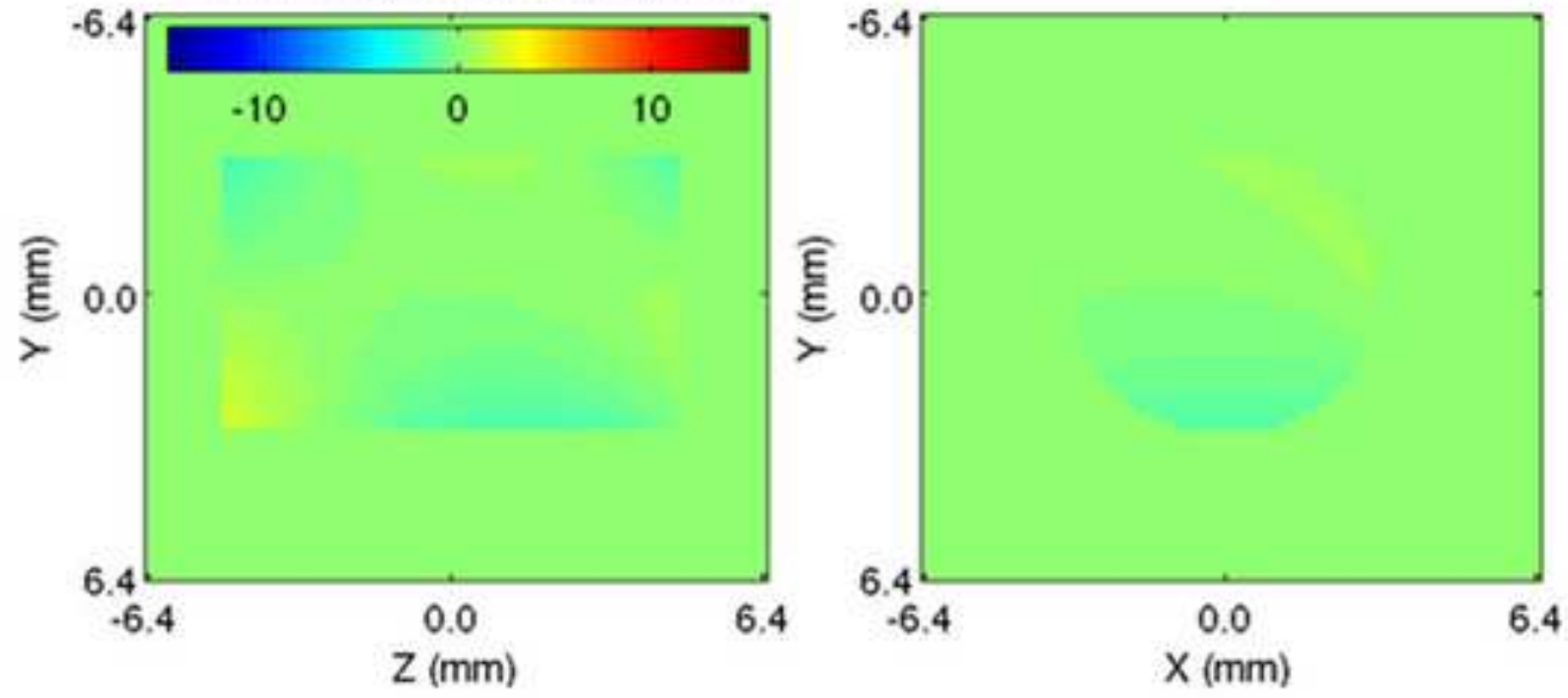

(b) 


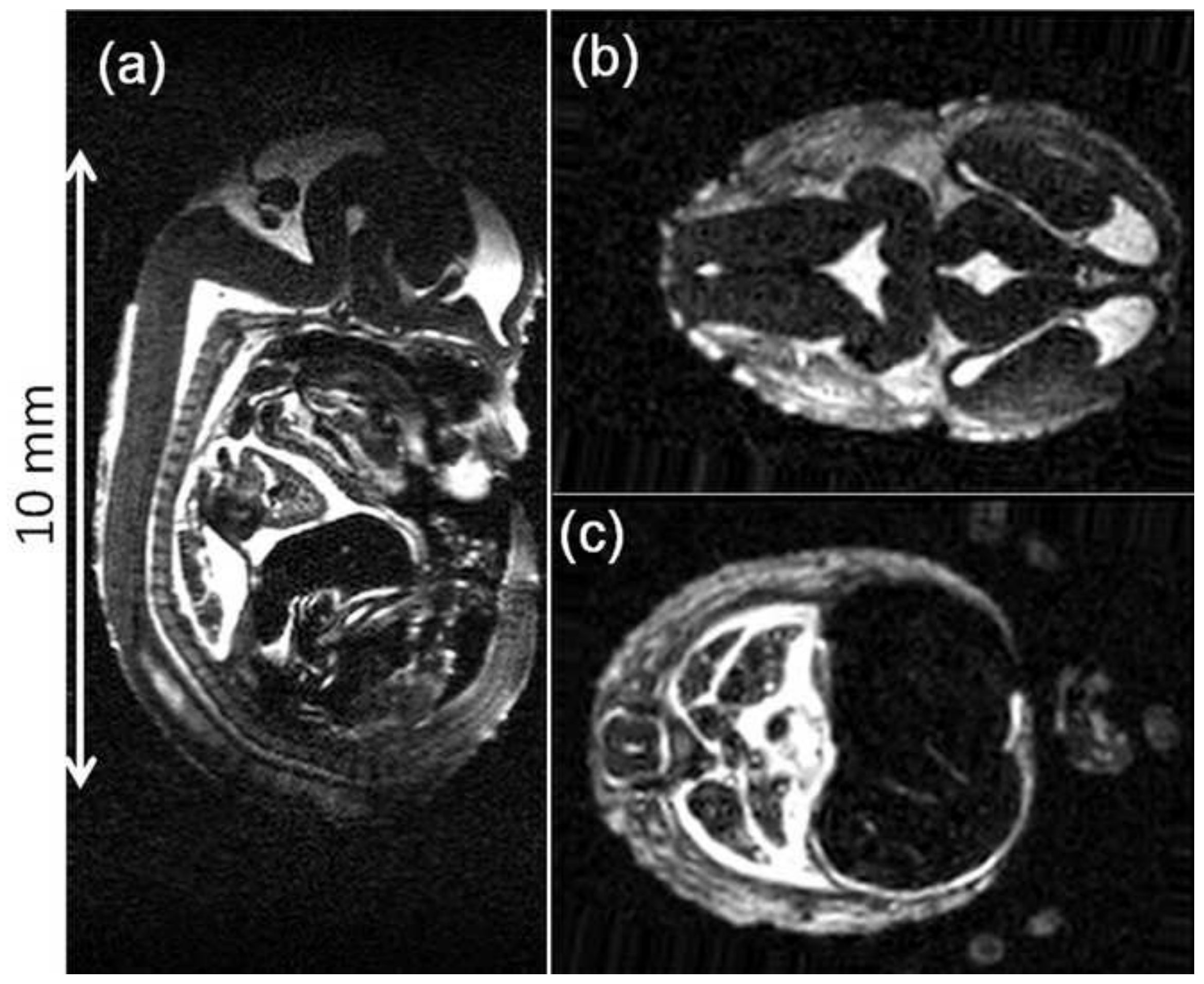




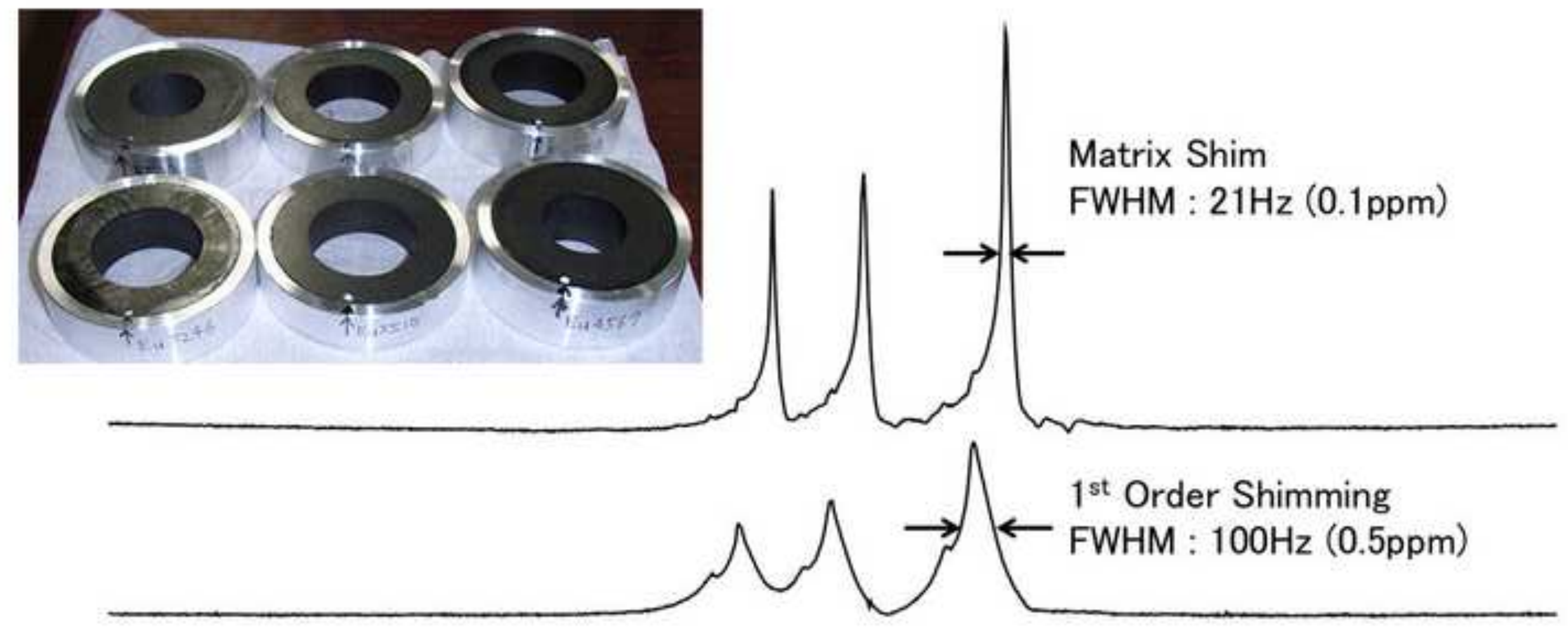

Without Shimming FWHM : 2979 Hz (15ppm)

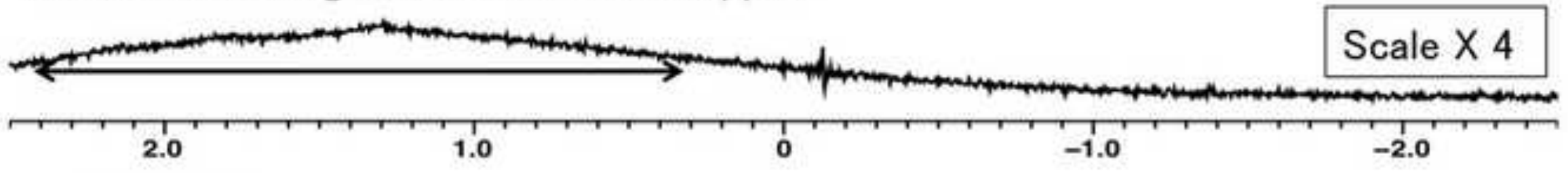

$1 \mathrm{H}$ : kilohertz 